\title{
Diferentes representações para o conceito de inequações: uma análise de livros didáticos de matemática do ensino médio
}

\author{
Representations different inequalities concept: an analysis of textbooks of high school \\ math
}

\author{
Veridiana Rezende \\ rezendeveridiana@gmail.com \\ Wilian Barbosa Travassos \\ wilians@hotmail.com.br
}

\begin{abstract}
Resumo
Apresentamos neste artigo uma análise, relacionada ao conceito de inequação e suas diferentes representações, realizada em seis (06) livros didáticos de Matemática do $1^{\circ}$ ano do Ensino Médio, aprovados pelo Programa Nacional do Livro Didático - PNLD 2015. Buscamos identificar as representações e os possíveis tratamentos e conversões priorizados pelos autores dessas obras, no que se refere especificamente às inequações de $1^{\circ}$ grau, inequação-produto, inequação-quociente e inequações simultâneas. Para as análises, consideramos todos os exercícios propostos nas referidas seções e suas respectivas respostas indicadas no final do livro didático do aluno. Como resultado, no que diz respeito aos tratamentos, destacamos que os livros didáticos priorizam o tratamento algébrico para a resolução das inequações. Com relação às conversões, nossas análises mostram que esta atividade cognitiva não é priorizada pelos autores. Uma das obras, por apresentar maior número de situações-problema, propicia a coordenação entre os registros língua natural e algébrico, fato que é positivo para o ensino de Matemática. Outra obra apresenta um maior número de registros gráficos, em relação às demais coleções, fato que pode enriquecer a aprendizagem do aluno.
\end{abstract}

Palavras-chave: Ensino de matemática; Representação semiótica; Inequações.

\begin{abstract}
We present in this article an analysis, related to the concept of inequality and its different representations, carried out in six (06) textbooks of Mathematics of the 1st year of High School, approved by the National Program of Didactic Book - PNLD 2015. We seek to identify representations and possible treatments and conversions prioritized by the authors of these works, specifically regarding first-degree inequalities, product-inequality, inequality-quotient and simultaneous inequalities. For the analyzes, we consider all the exercises proposed in the referred sections and their respective answers indicated at the end of the student textbook. As a result, with regard to treatments, we emphasize that textbooks prioritize algebraic treatment for the resolution of inequalities. Regarding the conversions, our analyzes show that this cognitive activity is not prioritized by the authors. One of the works, because it presents a greater number of problem situations, facilitates the coordination between the natural and algebraic language registers, a fact that is positive for the teaching of Mathematics. Another work presents a greater number of graphic records, in relation to the other collections, fact that can enrich the learning of the student.
\end{abstract}

Keywords: Mathematics teaching; Semiotic representation; Inequalities.

\section{Introdução}

Apresentamos neste artigo os resultados de uma investigação relacionada às seis coleções de livros didáticos do Ensino Médio aprovadas pelo Programa Nacional do Livro 
Didático - PNLD 2015. O PNLD é um programa do governo federal ${ }^{1}$ que propicia subsídios para o trabalho pedagógico do professor, por meio da distribuição de coleções de livros didáticos a todos os alunos de escolas públicas brasileiras. Os professores também recebem o manual do professor, que consiste do livro do aluno acrescidos de sugestões e comentários sobre a resolução das tarefas propostas, bem como sugestões de avaliações, metodologias, leituras, fundamentação teórica, todos de acordo com os objetivos educacionais propostos nos documentos curriculares.

A cada ano ocorre a avaliação de um segmento de ensino: anos iniciais do ensino fundamental, anos finais do ensino fundamental ou ensino médio. Com este procedimento que tem sido adotado pelo Ministério da Educação, garante-se que a cada três anos os livros sejam renovados nas escolas. Para o ano de 2015, foram aprovadas seis (06) coleções de livros didáticos de Matemática para o ensino médio.

Os livros recebidos devem ser conservados pelos alunos, e devolvidos no final do ano letivo para que possam ser utilizados por outras turmas nos dois anos seguintes. A cada ano, após as avaliações, é publicado no site do Ministério da Educação o Guia de Livros Didáticos (BRASIL, 2015) contendo os critérios de avaliações das obras, as resenhas das coleções aprovadas e discussões sobre características gerais das coleções aprovadas. Após a publicação do Guia, as coleções são enviadas para as escolas, para que a equipe pedagógica e professores de cada disciplina possam selecionar a coleção a ser adotada pela instituição.

Segundo os Parâmetros Curriculares Nacionais - PCN para a disciplina de Matemática (BRASIL, 1998), “o livro didático é um dos materiais de mais forte influência na prática de ensino brasileira" (p.96), e por esse motivo estes documentos alertam para a qualidade e à coerência desses materiais no que se refere aos objetivos educacionais.

Além dos objetivos educacionais, percebe-se a importância de se considerar as especificidades de cada disciplina no ato da escolha dos livros didáticos. A matemática, por exemplo, tem a especificidade de ser uma disciplina abstrata, e o acesso a seus objetos só ocorre por meio de representações.

Nesse sentido, o pesquisador francês Raymond Duval (2003) considera que o funcionamento do pensamento em Matemática apresenta certas especificidades em relação a outros domínios do conhecimento. Sobretudo, por ser uma ciência abstrata, é preciso considerar as diferenças existentes entre a atividade cognitiva em Matemática e de outras

\footnotetext{
${ }^{1}$ http://portal.mec.gov.br/pnld/apresentacao
} 
Ciências, tais como Biologia, Física ou Química, nas quais os conceitos podem ser observados ou manipulados por meios de experiências.

Desta forma, Duval (2011) defende que a compreensão de um conceito matemático ocorre por meio das diferentes representações; e, saber coordenar espontaneamente ao menos dois registros de representação pode ser um indicativo de que o aluno compreendeu o conceito em questão.

No que se refere às inequações, notamos que elas podem ser representadas por uma sentença matemática, contendo uma ou mais variáveis, definidas por sinais de desigualdade ou diferença entre os termos, estabelecendo sua representação algébrica. Além desta forma, podemos representa-la por meio de um enunciado na língua natural ${ }^{2}$, um gráfico, um segmento na reta numérica, entre outras.

Desse modo, considerando a importância do papel do livro didático para o ensino e para as aprendizagens em sala de aula, considerando a especificidade do conhecimento matemático, que, do ponto de vista de Duval, ocorre por meio de registros de representações, propomos neste artigo uma análise de seis livros didáticos de Matemática do Ensino Médio, aprovados pelo Programa Nacional do Livro Didático - PNLD $2015^{3}$, a respeito do conceito de inequação e suas diferentes representações.

\section{Registros de representação semiótica e o conceito de inequações}

Raymond Duval é filósofo e psicólogo de formação. Durante seus estudos no Instituto de Pesquisa sobre o Ensino de Matemática - IREM de Estrasburgo na França, desenvolveu uma teoria cognitiva denominada de Registros de Representação Semiótica.

Segundo Duval, as origens de sua teoria surgiram no momento em que, após anos de pesquisas sobre a compreensão de demonstrações de alunos do Collège $e^{4}$ e sobre a importância e a variedade das formas de linguagem, ele se deparou com a seguinte pergunta: "que tipo de esquema e, de modo mais geral, que tipo de representação é a mais pertinente para dar conta não apenas de um texto, mas de um raciocínio dedutivo, de uma argumentação, de uma escrita simbólica, etc.?” (FREITAS, REZENDE, 2013, p.13).

Em entrevista realizada por Freitas e Rezende (2013), Duval menciona que a principal dificuldade na aprendizagem da matemática deve-se ao fato de que os conhecimentos envolvidos não são descobertos nem explicados como os conhecimentos de outras áreas como

\footnotetext{
${ }^{2}$ Entendemos por língua natural a escrita em língua portuguesa - língua oficial do Brasil.

${ }^{3}$ Estes livros deverão permanecer vigentes nas escolas públicas nos anos de 2015, 2016 e 2017.

${ }^{4}$ Nível de ensino francês correspondente aos anos finais do Ensino Fundamental.
} 
física, botânica, geologia, etc. O pesquisador é enfático ao dizer que existem duas observações que permitem explicar o caráter cognitivo e epistemológico específico da matemática:

\footnotetext{
Em primeiro lugar, não existe acesso perceptivo, direto ou instrumental (microscópio, telescópio, osciloscópio, espectroscópio, etc.) aos números, às funções, às relações geométricas, ou seja, aos objetos matemáticos. Para termos acesso a esses objetos, precisamos de uma atividade de produção semiótica (FREITAS, REZENDE, 2013, p.16).
}

Segundo Duval, “[...] a originalidade da atividade matemática está na mobilização simultânea de ao menos dois registros de representação ao mesmo tempo, ou na possibilidade de trocar a todo o momento de registro de representação" (DUVAL, 2003, p.14).

Em sua teoria, Duval (2011) afirma que “[...] para que os alunos possam realmente compreender matemática [...] é preciso desenvolver outro tipo de funcionamento cognitivo que o praticado nas outras disciplinas" (2011, p.9). Como o acesso aos objetos matemáticos ocorre por meio de representações, Duval (2009) menciona sobre a importância de se explorar as diferentes representações de um conceito matemático.

Duval alerta sobre a necessidade de se trabalhar as diferentes representações de um conceito, pois, a estrita ligação a apenas uma representação poderá levar o aluno a confundir o próprio objeto matemático com sua representação. Mas, se os objetos matemáticos são abstratos, sendo necessários seus estudos por meio de representações, como não confundi-los com suas representações?

Essa imposição de condições leva a um paradoxo denominado por Duval de paradoxo cognitivo da matemática. Duval alega que “[...] é a possibilidade de multirrepresentação potencial de um mesmo objeto que permite contornar este paradoxo" (FREITAS, REZENDE, 2013, p.17). Esta é a condição fundamental para que o aluno saiba distinguir os objetos matemáticos de suas diferentes representações.

Na Matemática há uma grande variedade de representações semióticas, o enunciado de um problema, por exemplo, pode ser representado em língua natural, e suas resoluções podem vir na forma de cálculos algébricos, numéricos, gráficos, figural, entre outras. Duval (2003) classifica quatro grandes tipos diferentes de registros, são eles: a língua natural (associações verbais, conceituais, forma de raciocinar), os sistemas de escritas - simbólico (numéricas, algébricas), as figuras geométricas planas ou em perspectivas (operação operatória e não somente discursiva, construção com instrumentos) e os gráficos cartesianos (mudanças de sistemas de coordenadas, interpolação, extrapolação). 
Segundo Duval, os critérios de progressão na aprendizagem matemática são distintos do ponto de vista matemático e do ponto de vista cognitivo. O pesquisador entende que do ponto de vista matemático, compreender "é ser capaz de justificar um resultado por meio de uma propriedade" (FREITAS, REZENDE, 2013, p.20). Mas, do ponto de vista cognitivo, "é primeiro reconhecer o mesmo objeto em diferentes representações semióticas que podem ser feitas a partir dele, cujos conteúdos não têm nada em comum" (FREITAS, REZENDE, 2013, p.20).

Ao reconhecer diferentes representações de um mesmo objeto matemático, o aluno pode optar pelo modo mais econômico e mais eficaz para se resolver a tarefa proposta. Pois, "tendo mais registros, há um aumento potencial de possibilidades de trocas e, por conseguinte, há um aumento também na escolha mais econômica" (MORETTI, 2002, p.346).

Além disso, a importância das diferentes representações para a compreensão de um conceito também se dá pela complementariedade existentes entre os sistemas semióticos. Por exemplo,

[...] a linguagem discursiva não oferece as mesmas possibilidades que podem oferecer uma figura ou um diagrama. Isto quer dizer que de um ponto de vista cognitivo uma representação é parcial em relação aquilo que ela quer representar e que de um registro a outro não são os mesmos conteúdos de uma situação que são representados" (MORETTI, 2002, p.347).

No que se refere aos impactos das especificidades da matemática em relação aos processos de compreensão dos conceitos, Duval menciona que é preciso considerar as duas faces da atividade matemática, que ele as denomina de face exposta e a face oculta. A face exposta, segundo o pesquisador, corresponde aos objetos matemáticos (números, funções, equações, polígonos, poliedros, etc.), às suas propriedades, às fórmulas e algoritmos aos quais eles dão origem, às demonstrações, cujas aprendizagens são distribuídas ao longo de vários anos. Enquanto que a face oculta corresponde aos gestos intelectuais que constituem o caráter cognitivo e epistemológico específicos da matemática (FREITAS, REZENDE, 2013).

$\mathrm{O}$ ato de transitar de um registro de representação para outro é denominado por Duval como conversão. A conversão é o processo ao qual se realiza uma modificação externa ao registro representado, conservando a totalidade ou apenas uma parcela do conteúdo da representação inicial (DUVAL, 2012).

Para exemplificar as conversões relacionadas ao conceito de inequações, apresentamos o quadro 1 que se refere aos tipos de conversões que encontramos nas obras analisadas. Nesta 
pesquisa, denominamos por algébrica 1 a expressão algébrica inequação tal como $x+2>7$, e por algébrica 2 o conjunto solução de uma inequação: $S=\{x \in \mathbb{R} \mid x>5\}$.

Quadro 1: Conversões envolvendo inequações presentes nas coleções analisadas

Lingua Natural $\leftrightarrow$ Algébrica 1

Exemplo 1: "Para um atendimento domiciliar, um técnico em informática $X$ cobra $\mathrm{R} \$ 60,00$ a visita e $\mathrm{R} \$ 45,00$ a hora de trabalho; um técnico $Y$ cobra $R \$ 40,00$ a visita e $R \$ 50,00$ a hora de trabalho. A partir de quanto tempo de serviço é mais econômico contratar o técnico X?” (IEZZI et at, 2013, p.88).

R: Este problema pode ser resolvido por diversas maneiras, uma delas é por meio de uma inequação algébrica:

$$
\begin{gathered}
60+45 x<40+50 x \\
45 x-50 x<40-60 \\
-5 x<-20 \\
x>4
\end{gathered}
$$

Assim, realizamos a conversão Língua Natural para o Algébrico 1. A conversão Algébrico 1 para a Língua Natural, seria a interpretação do resultado em Língua Natural: A partir de 4 horas de serviço, é mais vantajoso a contratação do técnico X.

Algébrica $1 \rightarrow$ Gráfico

Exemplo 2: "Resolva graficamente a inequação $x+3>-6$ "(SMOLE, DINIZ, 2013, p.109).

R: Para este exemplo, consideramos $f(x)=\mathrm{x}+3$ e $g(x)=-6$, e construímos os gráficos de ambas as funções. Como queremos a parte positiva, ou seja, os valores tais que $f(x)>0$, a solução da inequação está representada em azul no gráfico.

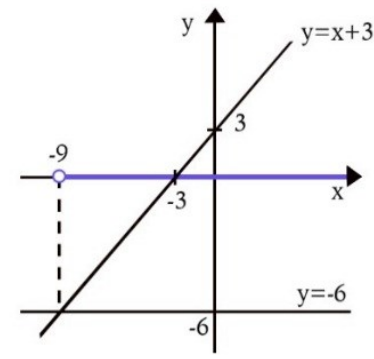

Tabular $\rightarrow$ Algébrica 1

Exemplo 3: "Três planos de telefonia celular são apresentados na tabela abaixo:

\begin{tabular}{|c|c|c|}
\hline Plano & Custo fixo mensal & Custo adicional por minuto \\
\hline $\mathrm{A}$ & $\mathrm{R} \$ 35,00$ & $\mathrm{R} \$ 0,50$ \\
\hline $\mathrm{B}$ & $\mathrm{R} \$ 20,00$ & $\mathrm{R} \$ 0,80$ \\
\hline $\mathrm{C}$ & 0 & $\mathrm{R} \$ 1,20$ \\
\hline
\end{tabular}

A partir de quantos minutos de uso mensal o plano A é mais vantajoso do que os outros dois?" (DANTE, 2013, p.88).

$\mathrm{R}$ : Para este caso, montamos as inequações algébricas conforme as informações do texto, considerando $\mathrm{x}$ como a quantidade de minutos, assim temos:

Plano A - B:

$$
\begin{gathered}
35+0,5 x<20+0,8 x \\
-0,3 x<-15 \\
x>50
\end{gathered}
$$

Plano A - C:

$$
\begin{gathered}
35+0,5 \mathrm{x}<0+1,2 \mathrm{x} \\
-0,7 \mathrm{x}<35 \\
\mathrm{x}>50
\end{gathered}
$$

Gráfico $\rightarrow$ Algébrica 2

Exemplo 4: "No plano cartesiano estão apresentadas os gráficos de duas funções, $f$ e $g$. 


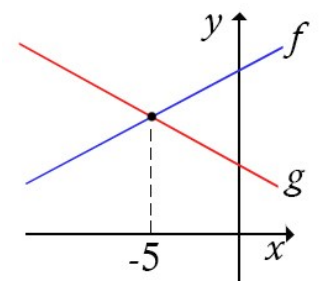

Qual é o conjunto solução da inequação $f(x)>g(x)$ ?" (LEONARDO, 2013, p.105).

$\mathrm{R}: \mathrm{S}=\{\mathrm{x} \in \mathbb{R} \mid \mathrm{x}>-5\}$

Gráfico $\rightarrow$ Língua Natural

Exemplo 5: "Sabendo que f e g são funções afins, analise o quadro de sinais abaixo usado para resolver uma inequação.

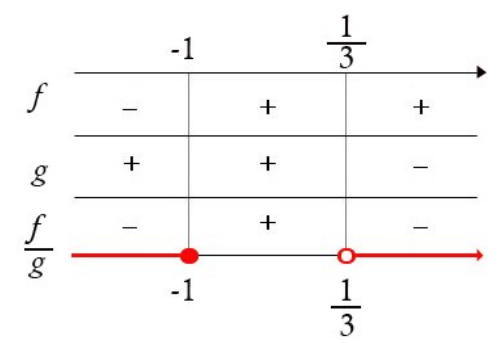

Esse quadro de sinais está sendo usado para resolver uma inequação-produto ou uma inequação-quociente?" (LEONARDO, 2013, p.107).

R: Inequação-quociente.

Figural $\rightarrow$ Algébrica 1

Exemplo 6: "No triângulo e no retângulo estão indicadas as medidas de seus lados.

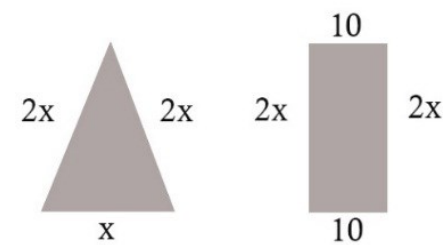

Qual é o maior valor inteiro que x pode assumir para que o perímetro do triângulo seja menor que o do retângulo?" (SOUZA, 2013, p.108)

$\mathrm{R}$ :

$$
\begin{gathered}
2 \mathrm{x}+2 \mathrm{x}+\mathrm{x}<2 \mathrm{x}+2 \mathrm{x}+10+10 \\
5 \mathrm{x}<4 \mathrm{x}+20 \\
x<20
\end{gathered}
$$

Logo, $x=19$.

Fonte: Autores

Todas as conversões do quadro 1 estão presentes nos livros analisados. No entanto, existem outras conversões relacionadas ao estudo das inequações que não foram encontradas 
em nossas análises, tais como: da representação gráfica para a numérica, da figural para numérica e da figural para a língua natural.

Além da conversão, existem as transformações que acontecem internamente ao registro. Essas modificações são denominadas por Duval como tratamento. Alguns exemplos de tratamento são: o cálculo numérico, que é um tipo de tratamento específico para expressões simbólicas; a paráfrase e a inferência, que são tratamentos no registro língua natural; a reconfiguração, que se aplica a figuras geométricas e a anamorfose que se aplica a toda representação figural (DUVAL, 2012).

A seguir, apresentamos um exemplo de tratamento presente nas obras analisadas.

Quadro 2: Tratamento

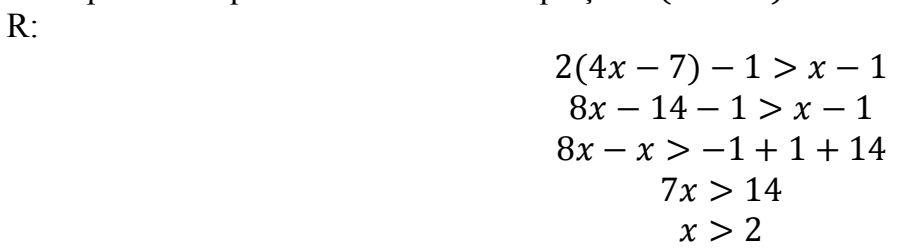

Fonte: autores

Segundo o pesquisador, a coordenação de ao menos dois registros de representação já é um indício para a apreensão conceitual de um objeto. É preciso que o objeto não seja confundido com sua representação, e que ele seja identificado em cada uma de suas diferentes representações. Nestes casos, a representação atua como sua verdadeira função, representar (DUVAL, 2012). A coordenação entre dois registros quaisquer ocorre por meio das operações conversão e tratamento (MORETTI, 2002, p.350).

A seguir, apresentamos os procedimentos metodológicos nos quais descrevemos o caminho seguido para as análises das obras, que estão seguidos das análises realizadas no que se refere às diferentes representações, possibilidades de tratamentos e conversões indicadas nas obras.

\section{Desenvolvimento e análises da pesquisa}

Nossas análises contemplaram as seis coleções aprovadas pelo PNLD 2015, sendo que cada coleção apresenta três volumes referentes aos $1^{\circ}, 2^{\circ}$ e $3^{\circ}$ anos do Ensino Médio. Para a nossa investigação, contemplamos a seção específica sobre inequações presente nos sumários do livro do aluno do $1^{\circ}$ ano, de cada coleção. 
As seis coleções analisadas estão especificadas no quadro 3, elas foram enumeradas de 1 a 6 , e no decorrer do texto serão referenciadas como LD, seguidas de um número de 1 a 6 .

Quadro 3: Obras analisadas e respectivas siglas adotadas

\begin{tabular}{|c|c|c|}
\hline Autores & Título da obra & Sigla livro didático \\
\hline Smole e Diniz & Matemática - Ensino Médio & LD1 \\
\hline Souza & Novo Olhar - Matemática & LD2 \\
\hline Paiva & Matemática PAIVA & LD3 \\
\hline Iezzi et al & Matemática - Ciências e Aplicações & LD4 \\
\hline Leonardo & Conexões com a Matemática & LD5 \\
\hline Dante & $\begin{array}{c}\text { Matemática - Contexto e } \\
\text { Aplicações }\end{array}$ \\
\hline
\end{tabular}

Fonte: Autores

No que se refere ao conceito de inequação, ao analisar as obras, consideramos as inequações do $1^{\circ}$ grau, inequações produtos de funções do $1^{\circ}$ grau, inequações quocientes de funções do primeiro grau e inequações simultâneas de funções do $1^{\circ}$ grau, conforme as definições apresentadas a seguir:

- Inequações do $1^{\circ}$ grau: Uma inequação do $1^{\circ}$ grau é toda sentença matemática envolvendo expressões algébricas com incógnitas de expoente 1, que, no caso de envolver apenas uma incógnita, é possível representa-la por uma das seguintes formas: $a x+b>0 ; a x+b<0 ; a x+b \geq 0 ; a x+b \leq 0$ e $a x+b \neq 0$, com $a$ e $b$ constantes reais e $a \neq 0$.

- Inequações de funções do $1^{\circ}$ grau - produto: Sendo $f$ e $g$ funções do $1^{\circ}$ grau na variável $x$, toda inequação que pode ser apresentada sob uma das formas abaixo é definida como inequação-produto: $f(x) \cdot g(x)>0 ; f(x) \cdot g(x)<0 ; f(x) \cdot g(x) \geq 0$; $f(x) \cdot g(x) \leq 0$ e $f(x) \cdot g(x) \neq 0$.

- Inequações de funções do $1^{\circ}$ grau - quociente: Sendo $f$ e $g$ funções do $1^{\circ}$ grau na variável $x$, toda inequação que pode ser apresentada sob uma das formas abaixo é definida como inequação-quociente: $\frac{f(x)}{g(x)}>0 ; \frac{f(x)}{g(x)}<0 ; \frac{f(x)}{g(x)} \geq 0 ; \frac{f(x)}{g(x)} \leq 0$ e $\frac{f(x)}{g(x)} \neq 0$ $(\forall x \in \operatorname{Dom}(g)$ tal que $g(x) \neq 0)$.

- Inequações do $1^{\circ}$ grau - simultâneas: Consistem em duas ou mais funções do $1^{\circ}$ grau, e podem ser representadas na forma de uma inequação dupla, tal como $f(x) \cdot g(x)$. $h(x)$ ou $\left\{\begin{array}{l}f(x) \cdot 0 \\ g(x) \cdot 0\end{array}\right.$ em que $\cdot$ pode significar $>, \geq,<$ ou $\leq$.

A coleta dos dados e as análises foram conduzidas de acordo com os seguintes critérios: i) Identificar a quantidade de exercícios sobre o conceito de inequações de $1^{\mathrm{o}}$ grau, 
inequações-produto, inequações-quociente e inequações simultâneas que cada obra traz, apresentando a relação dessa quantidade em exercícios fechados e situações-problema; ii) Identificar por meio da resolução apresentada no manual do professor os exercícios que utilizam apenas o tratamento como solução para a atividade; iii) Identificar as transformações entre representações presentes em cada livro, enfatizando as representações priorizadas pelos autores, sendo que algumas delas podem ser identificadas como conversões.

Embora Duval considere como essencial para a compreensão dos conceitos matemáticos a atividade cognitiva de conversão entre registros, ressaltamos que para as nossas análises também foram consideradas as transformações entre representações, mesmo que façam parte de um mesmo registro, tais como: transformação da representação algébrica para a numérica (e vice-versa), da representação algébrico 1 para a algébrico 2 (e vice-versa).

Entendemos por situações-problema atividades que consistem em exemplificar uma situação contextualizada, que leva o aluno a refletir e buscar meios para solucionar o problema. De acordo com Duval (2012), problemas propostos em língua natural são importantes para a aprendizagem matemática, pois favorecem conversão entre registros: “[...] esta conversão interna não é feita diretamente, ela passa por representações intermediárias" (DUVAL, 2012, p. 295). Ou seja, para o pesquisador, tarefas propostas em língua natural proporciona ao menos duas conversões, da língua natural para um outro registro, que pode ser simbólico (numérico ou algébrico), gráfico, figural, e a volta deste registro para a língua natural, que se trata da resposta para o problema solicitado.

Como exercícios fechados entendemos que são aqueles resolvidos por meio de cálculos, algoritmos ou aplicações de fórmula, sem nenhuma contextualização. Os exercícios fechados podem ou não proporcionar a conversão entre os registros.

Em nossas análises, observamos que alguns livros trazem exemplos/exercícios resolvidos explicando os processos de resolução para cada exercício, semelhante aos exercícios que são propostos em seu capítulo, são esses livros: LD-1 e LD-3, como mostra a figura 1. O livro LD-1 traz alguns exercícios cuja solução necessita a transformação do registro algébrico 1 para o registro gráfico, assim como também apresenta um exemplo semelhante ao exercício proposto, apresentado a seguir. 
Figura 1: Exemplo do livro didático Matemática - Ensino Médio

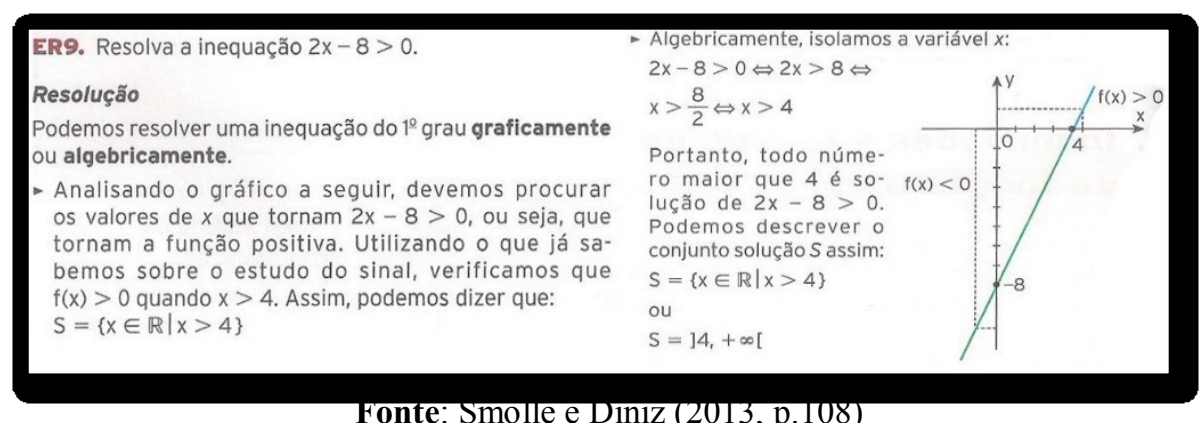

Fonte: Smolle e Dinız (2013, p.108)

Por outro lado, os livros LD-2, LD-4, LD-5 e LD-6 apresentam algum tipo de exercício proposto, cujo registro não é apresentado na introdução do conteúdo. Pudemos observar, também, que todos os livros trazem pelo menos um exercício proposto no qual é realizado a volta do registro, como o exemplo abaixo.

Figura 2: Exemplo do livro didático Matemática - Ensino Médio

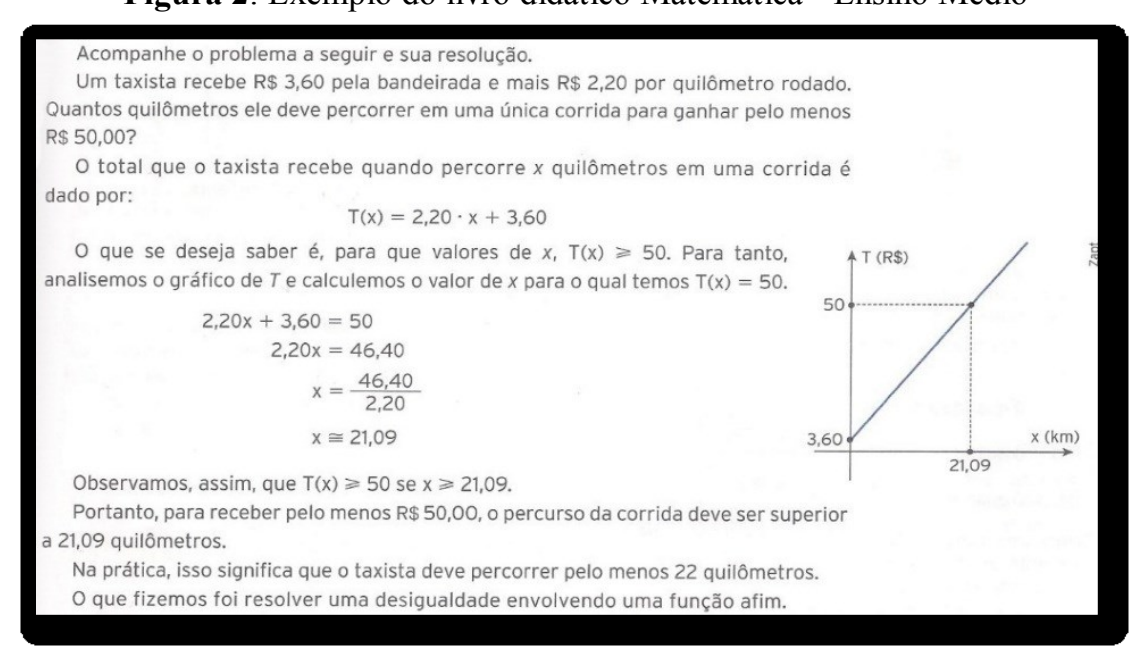

Fonte: Smolle e Diniz (2013, p.107)

Para Duval, este tipo de conversão que proporciona ida e volta entre os registros, neste caso língua natural - simbólico (algébrico) e simbólico (algébrico) - língua natural, é essencial para a aprendizagem dos conceitos. Entretanto, a conversão do registro língua natural para o registro simbólico (algébrico) e, na sequência, a conversão do simbólico (algébrico) para a língua natural, foi a única encontrada nos seis livros didáticos nos exercícios resolvidos. Além disso, destacamos que a resolução (figura 2) é enriquecida exibindo a representação gráfica da solução, fato que é positivo, do ponto de vista de Duval (2012).

Em relação às representação presentes nas obras, nossas análises indicaram seis representações diferentes, especificados a seguir: 
- Algébrica 1: Consideramos como representação algébrica 1 uma inequação representada como desigualdade ou diferença, do tipo $2 x-3>7$, ou $4 x-3 \neq 2-$ $x$.

- Algébrica 2: Consideramos a representação de um conjunto solução, restrito à um conjunto numérico. Exemplo: $S=\{x \in \mathbb{N} \mid x>12\}$.

- Gráfico: Consideramos como representação gráfica as funções ou figuras geométricas representadas no plano cartesiano, quadro de sinais e reta numérica.

- Língua Natural: Consideramos como representação em língua natural, exercícios ou respostas nas quais se utiliza da escrita em língua portuguesa. Exercícios em língua natural não necessariamente constituem-se como situações-problema.

- Numérica: Consideramos como representação numérica situações em que inequações são representadas por um intervalo numérico, por exemplo $S=] 2, \infty[$, ou apenas por números.

- Tabular: Consideramos como representação tabular, situações em que os dados principais do problema/resolução estejam dispostos em tabela, podendo ser realizado por meio de alguma condição (especificadas no exercício), a transformação destes dados para outro registro.

O quadro 4 disponibiliza o resultado encontrado em todas as obras analisadas, no que se refere a quantidade de situações-problema e exercícios fechados.

Quadro 4: Comparativo entre situações-problema e exercícios fechados

\begin{tabular}{|c|c|c|c|}
\hline \multicolumn{4}{|c|}{ Inequações do $1^{\circ}$ grau - PNLD 2015} \\
\hline Livros analisados & Situações - problema & Exercícios fechados & Total \\
\hline Livro Didático -1 & 6 & 10 & 16 \\
\hline Livro Didático -2 & 7 & 10 & 17 \\
\hline Livro Didático - 3 & 1 & 6 & 7 \\
\hline Livro Didático -4 & 4 & 13 & 17 \\
\hline Livro Didático -5 & 6 & 14 & 20 \\
\hline Livro Didático -6 & 6 & 6 & 12 \\
\hline \multicolumn{4}{|c|}{ Inequações produto e quociente - PNLD 2015} \\
\hline Livros analisados & Situações - problema & Exercícios fechados & Total \\
\hline Livro Didático - 1 & 0 & 13 & 13 \\
\hline Livro Didático - 2 & 0 & 0 & 0 \\
\hline Livro Didático - 3 & 1 & 9 & 10 \\
\hline Livro Didático - 4 & 0 & 20 & 20 \\
\hline Livro Didático -5 & 0 & 15 & 15 \\
\hline Livro Didático -6 & 0 & 7 & 7 \\
\hline \multicolumn{4}{|c|}{ Inequações simultâneas - PNLD 2015} \\
\hline Livros analisados & Situações - problema & Exercícios fechados & Total \\
\hline Livro Didático - 1 & 1 & 5 & 6 \\
\hline Livro Didático - 2 & 0 & 0 & 0 \\
\hline Livro Didático - 3 & 0 & 0 & 0 \\
\hline
\end{tabular}




\begin{tabular}{|c|c|c|c|}
\hline Livro Didático - 4 & 1 & 12 & 13 \\
\hline Livro Didático - 5 & 4 & 7 & 11 \\
\hline Livro Didático - 6 & 1 & 2 & 3 \\
\hline
\end{tabular}

Fonte: Autores

Com relação às inequações de grau 1, os livros trazem entre situações-problema e exercícios fechados, de 12 a 20 exercícios, com exceção do livro didático 3, que traz apenas 1 situação-problema e 6 exercícios fechados.

Observando o quadro das inequações-produto e inequações-quociente, é notado a carência de exercícios na forma de situação-problema e até mesmo exercícios fechados. O livro didático 2 não traz o conteúdo inequações-produto e inequações-quociente, e, apesar de o livro didático 1 trazer apenas um exercício fechado, ele também não explora tal conteúdo. $\mathrm{O}$ livro didático 3 foi o menos favorável no que se refere às inequações de $1^{\circ}$ grau, pois é o livro que apresenta a menor quantidade de exercícios envolvendo este conceito matemático. No entanto, é o único livro que apresenta uma situação-problema que envolve inequaçõesproduto e inequações-quociente, fato que consideramos positivo.

Para o conteúdo inequações simultâneas, os livros didáticos 2 e 3 trazem nada a respeito. As obras 1 e 6 trazem respectivamente 6 e 3 exercícios. Por outro lado, os livros didáticos 4 e 5 são os que trazem o maior número de exercícios, 13 exercícios o LD-4, sendo 12 exercícios fechados e uma situação-problema, e 11 exercícios o LD-5, sendo 7 exercícios fechados e 4 situações-problema. No total dos exercícios de inequações, os livros LD-4 e LD5 são os livros que contemplam o maior número de exercícios, sendo 50 exercícios (LD-4) e 46 exercícios (LD-5), enquanto que os livros LD-2 e LD-3 foram os que menos apresentam exercícios, sendo 17 exercícios.

Como segundo item das análises, identificamos dentre os exercícios aqueles em que, de acordo com o enunciado e a resposta no final do livro, espera-se que os alunos realizem somente o tratamento para solucioná-lo, conforme quadro 5.

Quadro 5: Tratamentos relacionado à inequações

\begin{tabular}{|c|c|c|c|}
\hline \multicolumn{2}{|c|}{ Tratamento - Inequações ( $1^{\circ}$ grau, produto, quociente e simultâneas) } \\
\hline Livros didáticos & Inequações & Representação & Exercícios \\
\hline Livro Didático - 1 & Inequação do $1^{\circ}$ grau & Algébrica 1 & 2 \\
\hline Livro Didático - 2 & Inequação do $1^{\circ}$ grau & Algébrica 1 & 1 \\
\hline \multirow{2}{*}{ Livro Didático - 3 } & Inequação Produto & Algébrica 1 & 1 \\
\cline { 2 - 4 } & Inequação Quociente & Algébrica 1 & 1 \\
\hline Livro Didático - 4 & Inequação Produto & Algébrica 1 & \\
\hline
\end{tabular}




\begin{tabular}{|c|c|c|c|}
\hline \multirow{3}{*}{ Livro Didático - 5 } & Inequação do $1^{\circ}$ grau & Algébrica 1 & 1 \\
\cline { 2 - 4 } & Inequação Produto & Algébrica 1 & 3 \\
\cline { 2 - 4 } & Inequação Quociente & Algébrica 1 & 1 \\
\cline { 2 - 4 } & Inequação Simultânea & Algébrica 1 & 2 \\
\cline { 2 - 4 } & Inequação do $1^{\circ}$ grau & Algébrica 1 & 3 \\
\hline
\end{tabular}

Fonte: Autores

Nota-se que apenas o livro LD-5 contempla o tratamento numérico além da representação algébrica 1, sendo que os demais livros trazem apenas exercícios algébricos em que se é possível resolvê-los por meio de transformações internas em relação à representação algébrica 1. Os livros LD-1, LD-2 e LD-6 apresentam tratamento nos exercícios proposto apenas para o conceito inequações do $1^{\circ}$ grau; o livro LD-4 apenas para inequações produto; o livro LD-3 inequações produto e quociente, já o livro LD-5 apresenta pelo menos um exercício para cada conteúdo de inequações em que estamos abordando. Diante deste quadro, podemos notar que os autores priorizam, exclusivamente, o tratamento algébrico em suas obras, em se tratando das inequações.

A seguir é apresentado o quadro referente às transformações entre representações, sendo que algumas delas indicam possibilidades do processo de conversão, presentes nos exercícios dos seis livros didáticos. Informamos que tais transformações foram consideradas a partir dos enunciados e das respostas indicadas no final do livro do aluno para cada um destes exercícios. Por exemplo, no exercício: Resolva a inequação $\mathrm{x}-2>0$, alguns livros apresentam como resposta na forma de um intervalo numérico: $S=(2, \infty)$, outros livros a resposta apresentada está na forma de conjunto solução: $S=\{x \in \mathbb{R}=\{x>2\}$, embora existem várias possibilidades de resposta, consideramos aquela indicada no livro para a conferência do aluno (e do professor).

Quadro 6: Transformação entre representações - PNLD 2015

\begin{tabular}{|l|c|c|c|c|c|c|}
\hline \multicolumn{7}{|c|}{ Inequações do $1^{\circ}$ grau } \\
\hline \multicolumn{1}{|c|}{ Transformações } & LD-1 & LD-2 & LD-3 & LD-4 & LD-5 & LD-6 \\
\hline Algébrica 1 $\rightarrow$ Algébrica 2 & - & 4 & 3 & 9 & 5 & 2 \\
\hline Algébrica 1 $\rightarrow$ Gráfico & 4 & - & - & - & - & - \\
\hline Algébrica 1 $\rightarrow$ Língua Natural & 2 & 11 & 1 & 3 & - & 3 \\
\hline Algébrica 1 $\rightarrow$ Numérica & 4 & 1 & 3 & 3 & - & - \\
\hline
\end{tabular}




\begin{tabular}{|c|c|c|c|c|c|c|}
\hline Gráfica $\rightarrow$ Algébrica 1 & - & - & - & 1 & 6 & - \\
\hline Gráfica $\rightarrow$ Algébrica 2 & - & - & - & - & 3 & - \\
\hline Figural $\rightarrow$ Algébrica 1 & - & 1 & - & - & - & - \\
\hline Língua Natural $\rightarrow$ Algébrica 1 & 6 & 8 & 1 & 4 & 6 & 6 \\
\hline Tabular $\rightarrow$ Algébrica 1 & - & 1 & - & - & - & 1 \\
\hline \multicolumn{7}{|c|}{ Inequações - produto } \\
\hline Transformações & LD-1 & LD-2 & LD-3 & LD-4 & LD-5 & $\overline{\mathrm{LD}-6}$ \\
\hline Algébrica $1 \rightarrow$ Algébrica 2 & 4 & - & 3 & 8 & 3 & 3 \\
\hline Algébrica $1 \rightarrow$ Língua Natural & - & - & - & 1 & - & - \\
\hline \multicolumn{7}{|c|}{ Inequações - quociente } \\
\hline Transformações & LD-1 & LD-2 & LD-3 & LD-4 & LD-5 & LD-6 \\
\hline Algébrica $1 \rightarrow$ Algébrica 2 & 9 & - & 4 & 11 & 4 & 4 \\
\hline Língua Natural $\rightarrow$ Algébrica 1 & - & - & 1 & - & - & - \\
\hline Língua Natural $\rightarrow$ Algébrica 2 & - & - & 1 & - & - & - \\
\hline Gráfica $\rightarrow$ Algébrica 1 & - & - & - & - & 1 & - \\
\hline Gráfica $\rightarrow$ Algébrica 2 & - & - & - & - & 1 & - \\
\hline Gráfica $\rightarrow$ Língua Natural & - & - & - & - & 1 & - \\
\hline \multicolumn{7}{|c|}{ Inequações simultâneas } \\
\hline Transformação & LD-1 & LD-2 & LD-3 & LD-4 & LD-5 & LD-6 \\
\hline Algébrica $1 \rightarrow$ Algébrica 2 & 5 & - & - & 12 & 4 & 2 \\
\hline Algébrica $1 \rightarrow$ Língua Natural & 1 & - & - & 1 & 4 & - \\
\hline Língua Natural $\rightarrow$ Algébrica 1 & 1 & - & - & 1 & 4 & 1 \\
\hline Gráfica $\rightarrow$ Algébrica 2 & - & - & - & - & 1 & - \\
\hline
\end{tabular}

Fonte: Autores

Destacamos que de acordo com os quatro tipos de registros propostos por Duval: língua natural, simbólico (numérico, algébrico), gráfico e figural, dentre as transformações entre representações indicadas no quadro 6 , consideramos como possibilidade de conversão as seguintes transformações: algébrica $\leftrightarrow$ gráfica; algébrica $\leftrightarrow$ língua natural; gráfica $\rightarrow$ algébrica; figural $\rightarrow$ algébrica.

No que se refere às transformações entre representações, percebemos, de acordo com o quadro 6, que nas obras analisadas a mudança entre representações não é priorizada, particularmente, podemos afirmar que a conversão não é priorizada. No entanto, algumas obras indicam mais possibilidades de transformação entre representações relacionadas às inequações do que as demais. Notamos, por exemplo, que a mudança da representação 
algébrica 1 para a algébrica 2 é priorizada pela coleção 4 . O livro didático 5 se destaca perante os demais por propiciar a conversão do gráfico para a representação algébrica 1 , fato que não apareceu nas demais obras analisadas.

\section{Conclusões}

As análises desta pesquisa mostram os diferentes tipos de representações relacionadas ao conceito de inequação que estão contempladas nas obras do PNDL 2015, quando se trata de introduzir este conceito aos alunos no $1^{\circ}$ ano do Ensino Médio. Além disso, mostramos as prioridades atribuídas pelos autores das obras no que se refere às situações - problema, tratamentos, transformações entre representações e possibilidades de conversões, no que concerne aos enunciados dos exercícios propostos e as respectivas respostas indicadas no final do livro didático.

No que se refere aos tratamentos, notamos em nossas análises a prioridade atribuída pelos livros ao tratamento algébrico para a resolução dos exercícios propostos. Realizar tarefas envolvendo tratamento algébrico é importante para que os alunos possam compreender sobre o desenvolvimento e propriedades das inequações. No entanto, o tratamento envolvendo outros registros também é importante durante a aprendizagem desse conceito matemático, fato que não foi percebido nas obras, com exceção da obra 5 que apresentou o tratamento numérico além do tratamento algébrico.

Destacamos como ponto positivo na coleção 2 o fato de contemplar o maior número de situações-problema dentre as obras analisadas, oportunizando ao aluno trabalhar com no mínimo dois registros, língua natural e algébrico, por exemplo. Além disso, as situaçõesproblema, geralmente, solicitam a resposta final em língua natural, propiciando a ida e a volta da conversão, fato que Duval (2012) considera importante para a aprendizagem em Matemática. Porém, esta obra não explora o conteúdo inequação-produto, inequaçãoquociente e inequações simultâneas.

Já a transformação da representação algébrica 1 para a representação algébrica 2 é priorizada pela coleção 4 . O livro didático 5 se destaca perante os demais por propiciar a conversão do registro gráfico para o registro simbólico (algébrico 1), fato que pode enriquecer a compreensão do aluno, a respeito do conceito de inequação. Além disso, em um dos livros (LD-6) encontramos erros na resolução proposta, o exercício (DANTE, 2014, p.84-85) referese à compra e vendas de maçãs, que se trata de um conjunto discreto, no entanto, a resolução do problema é realizada utilizando o conjunto dos números reais, no lugar dos números 
naturais. Consideramos que esse é mais um dos motivos para a importância de um olhar crítico para as obras adotadas.

As análises das obras, que estão em vigor nas escolas públicas brasileiras entre os anos de 2015 e 2017, mostram a carência da coordenação (tratamento e conversão) entre os registros de representação semiótica referente ao conceito de inequação. Além disso, mostram as diferenças existentes entre as obras no que se refere à exploração, tratamento e conversão entre registros.

Assim, considerando as diferenças apontadas em nossas análises no que concerne aos registros de representação, as transformações entre representações e possibilidades de conversões identificados nas obras, alertamos para a importância do momento da escolha do livro didático pelos professores. Pois, se o livro ainda é o principal instrumento para auxiliar a prática pedagógica do professor em sala de aula, e se as coleções apresentam características distintas, é imprescindível que a sua escolha seja cautelosa no sentido de atender as especificidades do ensino de matemática, uma vez que, conforme mostramos com esta pesquisa, as obras apresentam diferenças entre si, no que se refere aos registros de representação semióticas contemplados em cada obra.

Do ponto de vista da compreensão de conceitos matemáticos, nossas análises mostram a importância de o professor complementar a preparação de suas aulas com outros livros didáticos e materiais, propiciando aos alunos experiências com tarefas matemáticas que visam propiciar a articulação entre diferentes representações e diferentes registros. Pois, segundo Duval, este é o caminho para levar os alunos a reconhecer o mesmo objeto matemático (abstrato) em suas diferentes representações, culminado com a compreensão dos conceitos estudados.

\section{Referências}

BRASIL. Guia de Livros Didáticos PNLD 2015. Ministério da Educação. Secretaria da Educação Básica. Brasília, 2015.

BRASIL. Parâmetros Curriculares Nacionais: Matemática. Ministério da Educação. Secretaria de Educação. Brasília, 1998.

DANTE, L. R. Matemática Contexto e Aplicações. 2a Edição. São Paulo: Ática, 2014. Volume I.

DUVAL, R.; FREITAS, J. L. M.; REZENDE, V. Entrevista: Raymond Duval e a Teoria dos Registros de Representação Semiótica. Revista Paranaense de Educação Matemática, v. 2, p. 10-34, 2013.

DUVAL, R. Registros de Representação Semiótica e Funcionamento Cognitivo do pensamento. Revemat. Florianópolis, v. 07, n. 2, p.266-297, 2012. 
DUVAL, R. Ver e ensinar a matemática de outra forma: entrar no modo matemático de pensar os registros de representações semióticas. Org. Tânia M.M. Campos. São Paulo: PROEM, 2011.

DUVAL, R. Registros de representação semiótica e funcionamento cognitivo da compreensão em matemática. In: MACHADO, S. (Org). Aprendizagem em matemática: registros de representação semiótic $a$. Campinas: 2003, p.11-33.

IEZZI, G; DOLCE, O; DEGENSZAJN, D; PÉRIGO, R; ALMEIDA, N. Matemática Ciência e Aplicações. $7^{a}$ Edição. São Paulo: Saraiva, 2013. Volume I.

MARTINS DE LEONARDO, F. Conexões com a Matemática. $2^{\text {a }}$ Edição. São Paulo: Moderna, 2013. Volume I.

MORETTI, M. T. O papel dos registros de representação semiótica na aprendizagem de matemática. Contrapontos. Ano 2, n.6, p.423 - 437, Itajaí/SC, 2002.

PAIVA, M. Matemática Paiva. 2a Edição. São Paulo: Moderna, 2013. Volume I.

SMOLE, K. T; DINIZ, M. I. Matemática Ensino Médio. $8^{\text {a }}$ Edição. São Paulo: Saraiva, 2013. Volume I.

SOUZA, J. Novo Olhar Matemática. 2a Edição. São Paulo: FTD, 2013. Volume I. 